\title{
Spatiotemporal Characteristics and Resilience of Urban Network Structure during the Spring Festival Travel Rush: A Case Study of Urban Agglomeration in the Middle Reaches of Yangtze River in China
}

\author{
Shimei Wei $\mathbb{D}$ and Jinghu Pan \\ College of Geography and Environmental Science, Northwest Normal University, Lanzhou 730070, China \\ Correspondence should be addressed to Jinghu Pan; panjh_nwnu@nwnu.edu.cn
}

Received 5 May 2021; Revised 26 August 2021; Accepted 7 September 2021; Published 28 September 2021

Academic Editor: Alejandro F. Villaverde

Copyright ( $(2021$ Shimei Wei and Jinghu Pan. This is an open access article distributed under the Creative Commons Attribution License, which permits unrestricted use, distribution, and reproduction in any medium, provided the original work is properly cited.

\begin{abstract}
With the increasing trend of globalization, large-scale and diffuse population flow have become vital carriers characterizing users' spatial behaviors. Network analysis provides a new perspective to uncover the topology and evolution of the population flow and understand its influence on regional development. By gathering the Autonavi migration index during the Spring Festival travel rush (SFTR) in 2019, 2020, and 2021, the population flow networks among 31 cities of urban agglomeration in the middle reaches of the Yangtze River were constructed to analyze spatiotemporal dynamic characteristics and explore the structure resilience. Results show that although the changing trends of population flow during the 40-day SFTR of 2019, 2020, and 2021 are consistent, the population floating scale in 2020 and 2021 shows remarkable abnormalities before and after the Spring Festival due to the need for prevention and control of COVID-19. The intensity of population floating of the regional urban network in 2020 was the weakest, and Changsha became the focus of most population flow, while Wuhan was the most advantageous city in 2019 and 2021. As the third core city in the regional network, the siphon effect of Nanchang was still weak. A situation of tripartite confrontation in the region is formed. However, the higher intensity of population flow in 2021 increased the instability of the regional urban network, potentially exposing the region to higher risks and pressures. Therefore, it is necessary to pay more attention to the peripheral cities to improve regional resilience.
\end{abstract}

\section{Introduction}

With the increasing trend of globalization and the promotion of technology, the improvement in high-speed connection infrastructures (aviation, high-speed rail, telecommunications, Internet, etc.) has strengthened the connections between cities. The hierarchical space of cities dominated by administrative divisions tends to change into the functional space of the relational network. Especially in the recent years, with the rapid development of society and economy, the connections among cities tend to be complicated and diversified, and the dynamic urban network in economy, technology, and information-driven by population flow has become a hot research topic. The "space of flows" theory proposed by Castells [1] can describe vividly the phenomenon of spatial connection in city networks. The urban network is usually represented by a network diagram, which is composed of nodes and links (edges). In a regional urban network, nodes and links correspond to cities and connections between cities, respectively. Flow elements, population flow, information flow, capital flow, technology flow, etc., gradually get rid of the limitation of geographical space, and communicate and propagate in larger regions. With the rise of flow space theory, regional urban networks were constructed based on the data of various resource flows among cities, and the special characteristics of their spatial structure were studied, which provide scientific support for the strategies of regional development. 
As one of the largest and most far-reaching geographic processes since China's reform and opening up, population migration, which is an important carrier of flow elements among cities, is regarded as an activity in which essential productive factors are reconfigured in space $[2,3]$. The Chinese Lunar New Year, the Spring Festival, is the most celebratory time of the year in China, during which a massive human migration takes place as individuals travel back to their hometowns [4]. China's Spring Festival travel rush (SFTR) is described as rarely seen population floating in the world and is the largest and periodically floating population in human history due to the particularity of its population flow scale. National Development and Reform Commission of China stated that the annual spring migration (called Chunyun in China) often lasts for 40 days. During the SFTR in 2019, 2020, and 2021 (from January 21, 2019 to March 1, 2019; from January 10, 2020 to February 18, 2020; from January 28, 2021 to March 8, 2021), there had been about 3 billion, slightly higher than 3 billion, and 1.7 billion passengers between urban and rural areas, respectively. The large-scale and diffuse population flow promotes the reaggregation and diffusion of social and economic factors to some extent. Meanwhile, it is, however, a challenge and examination to the transportation infrastructure systems [5].

It is generally known that the Corona Virus Disease 2019 (COVID-19) was first identified and reported in Wuhan of China, the capital city of Hubei province with 11 million inhabitants and the most significant transport hub in Central China [6]. Due to its characteristics of person-to-person contact, extensive population flow has substantially increased social contacts in public, which caused COVID-19 to reach essentially everywhere [7]. On December 31, 2019, before the Chinese Lunar New Year (began on January 24, 2020), about 5 million people left Wuhan [7]. Further spatial spread of COIVD-19 was of great concern because of the upcoming Spring Festival Holiday (from January 24, 2020 to January 31, 2020). To control the rapid spread of COVID-19, Chinese government ordered lockdown policies in late January 2020 [8]. Particularly in Wuhan, where the largest number of infected people live, the Municipal Government completely closed the city on January 23, 2020. Nevertheless, the global multipoint outbreak of COVID-19 has evolved from a public health emergency into a nontraditional disaster. It directly exposes that without the support of a solid healthy and safe urban development environment, the urban prosperity and vitality may strengthen the vulnerability of the city, and even cause short-term "shock" and long-term "sequelae" of the city. Additionally, in the process of coping with the COVID-19, China's highly effective coordination mechanism among cities, nodes control of urban traffic network, construction of interregional public health networks, and regional coordinated production of antiepidemic materials, to a certain extent, reflect the coordinated cooperation among cities in response to disasters and crises, which will form a benign network synergy effect. Therefore, faced with long-term chronic pressures and short-term impacts, the underlying questions are how to investigate spatiotemporal characteristics of population mobility networks and how to measure the capacity of regional resilience.

Regional resilience is a rising concept in international research fields. It is usually measured by urban network structure resilience, focusing on the capacity of a city system to restore, maintain, or improve the original network characteristics and important functions in response to regional shocks [9]. With the acceleration of the process of urbanization, the uncertainties and unknown risks facing urban systems are also increasing [10]. Especially in the recent years, the sustainable development of cities has been seriously threatened by natural disasters, human disasters, the economy, the environment, safety, transportation, and society, which directly affect the life safety and quality of life of urban residents. In these cases, a system can fail, leading to a major reduction or complete loss in performance with respect to some or all measures. Moreover, if the function of a city fails, other cities in the region may no longer operate normally, but consider and select new connection objects. Accordingly, the pressures of other cities will increase, which bring some challenges to the sustainable development of regions. As a typical embodiment of regional spatial characteristics, the urban network structure has an impact on regional sensitivity to shocks, adaptability, and the ability to develop new growth paths [11]. Since Holling (1973) [12] first introduced the concept of resilience into ecosystem studies, it has been routinely used in research in disciplines ranging from environmental research to materials science and engineering, psychology, sociology, and economics $[13,14]$. Recent studies have shown that the concept and definition of resilience can also be applied to regional networks [15]. In previous studies on the resilience of regional networks, some scholars focused on the resilience characteristics of finance networks [16], infrastructure networks [17], transport networks [18, 19], and so forth. Although still gathering momentum, the previous studies mainly focus on theoretical exploration [20]. However, in the face of external shocks, especially against the backdrop of the enormous negative impact of the COVID-19 on the global economy, the assessment of the resilience of the urban network structure is of great and far-reaching significance to enhance urban abilities to prevent system failures and to make contingency plans and promote the high quality and sustainable development of the region.

As the main carrier of flow elements, intercity population migration pushes the rapid flow and optimization of production factors within a region. Therefore, urban population migration networks based on geospatial "flow data" have attracted increasing attention of scholars in the recent years. Some earlier studies mainly used static data, such as census data and population sampling survey data, which cannot reveal the increasingly complex interaction between cities from the perspective of space of flows [21]. As mobile Internet has found its way into people's everyday life, the global positioning system (GPS), location-based services (LBSs), and location sharing services are increasingly used to discover the geographic locations of users and their individual preferences, travel routes, activity trails, and social networks, thereby elucidating their daily spatiotemporal 
behavior [3]. LBSs can provide accurate information such as starting points, ending points, and even individual spatial trajectories. The association between regional entities can be visualized as a variety of practical measurable association flows. Accordingly, related studies based on various big data are in full swings, such as microblog check-in [22], Tencent migration [3], Baidu migration data [23], and Facebook [24]. From the point of view of the research time-period, many studies took the public holidays as examples, such as China's Spring Festival, the National Day, and the Mid-Autumn Festival travel rush, the daily time-period was also involved $[3,22,25,26]$. In addition, the research contents have included population flow intensity, scale, spatial distribution, travel pattern, etc. The spatial scale of related researches has involved from global to local cities. Overall, multiperspective and multiscale research on population flow networks is becoming an important way to understand the complex and dynamic spatial relationships between cities based on LBS data. However, the existing literature on population flow networks is mostly focused on a certain year, lacking vertical comparison from the perspective of evolution. Second, although directed weighted population flow networks were constructed, the directionality of population flow has been neglected in the analysis process, thereby leading to the underestimation or overestimation of the asymmetric relationship between urban nodes. The above illustrates that in the era of big data with rapid urban developments, the spatial patterns and scale characteristics of urban networks have already changed from static to complex spatiotemporal dynamics. Therefore, it is necessary to study the dynamic pattern characteristics of urban networks and their resilience based on spatiotemporal big data. In addition, Autonavi Map (AMap) application records population flow routes using its LBS device in real-time, dynamically, completely, and systematically, producing Autonavi migration big data. The available data period is from June 2018 to present, which makes it possible to characterize the population flow during the SFTR in different periods.

From the national strategy to promote the Yangtze River Economic Belt, urban agglomeration in the middle reaches of the Yangtze River plays a vital role in China's regional development pattern because it is an important support to promote the coordinated development of the Yangtze River Economic Belt. Moreover, Wuhan, a core city of the urban agglomeration, served as the high-risk area for the outbreaks of COVID-19 during the SFTR in 2020. The large-scale population flow among regional cities, especially within the urban agglomeration, further exacerbated the spread of COVID-19. It is, therefore, necessary to clearly understand the population flow between cities within the region, determine its spatiotemporal characteristics, and measure its network structure resilience. Taking 31 cities of the urban agglomeration in the middle reaches of Yangtze River as the research subjects, through gathering the Autonavi migration big data during the SFTR in 2019, 2020, and 2021, the spatiotemporal complex characteristics of the urban network of urban agglomeration in the middle reaches of the Yangtze River are revealed herein from the perspective of population flow. Then, the structural resilience of the regional urban networks is estimated when perturbations or major events occurred. Furthermore, it is expected to put forward specific strategies and suggestions in combination with the research conclusions to promote the region for further development. This study not only provides a new research perspective and methodological reference for research on urban networks, but also contributes to further regional coordination and sustainable development.

\section{Study Area}

The urban agglomeration in the middle reaches of the Yangtze River, also known as the "Triangle of Central China”, is a super large national urban agglomeration located in the middle reaches of the Yangtze River, mainly formed by the Wuhan metropolitan area, ChangshaZhuzhou-Xiangtan city group, and Poyang Lake city group. In this study, 31 cities defined in the development plan of urban agglomeration in the middle reaches of the Yangtze River issued by the National Development and Reform Commission in April 2015 were selected as the research objects (Figure 1). Specifically, the study area includes 13 cities in the Hubei province (Wuhan, Huangshi, Ezhou, Huanggang, Xiaogan, Xianning, Xiantao, Qianjiang, Tianmen, Xiangyang, Yichang, Jingzhou, and Jingmen), 8 cities in the Hunan province (Changsha, Zhuzhou, Xiangtan, Yueyang, Yiyang, Changde, Hengyang, and Loudi), and 10 cities in the Jiangxi province (Nanchang, Jiujiang, Jingdezhen, Yingtan, Xinyu, Yichun, Pingxiang, Shangrao, Fuzhou, and Jian), which covers a total area of approximately $31.7 \times 10^{4} \mathrm{~km}^{2}$. Among them, Wuhan, Changsha, and Nanchang, on the one hand, as provincial capitals, lead the social and economic development of the Wuhan metropolitan area, Changsha-Zhuzhou-Xiangtan city group, and Poyang Lake city group, respectively. On the other hand, the three core cities of the urban agglomeration form a "tripartite confrontation" spatial structure and play important dispersion and aggregation functions in the region. At the end of 2019, the regional GDP was 9.3833 trillion yuan, and the total resident population was 130.6491 million. As an important part of the Yangtze River Economic Belt, the urban agglomeration in the middle reaches of the Yangtze River not only connect the eastern and western regions, connect the South and the North, but also are the central area to implement the strategy of promoting the rise of the central region, comprehensively deepening the reform and opening up and promoting the new urbanization. What's more, the urban agglomeration is positioned as a new growth pole for China's economy, a leading area for new urbanization in the central and western regions, a demonstration area for inland open cooperation, and a leading area for "two oriented" social construction. Therefore, it plays an important role in China's regional development pattern.

\section{Data and Methods}

3.1. Data Sources. Taking 31 cities of the urban agglomeration in the middle reaches of the Yangtze River as examples, 

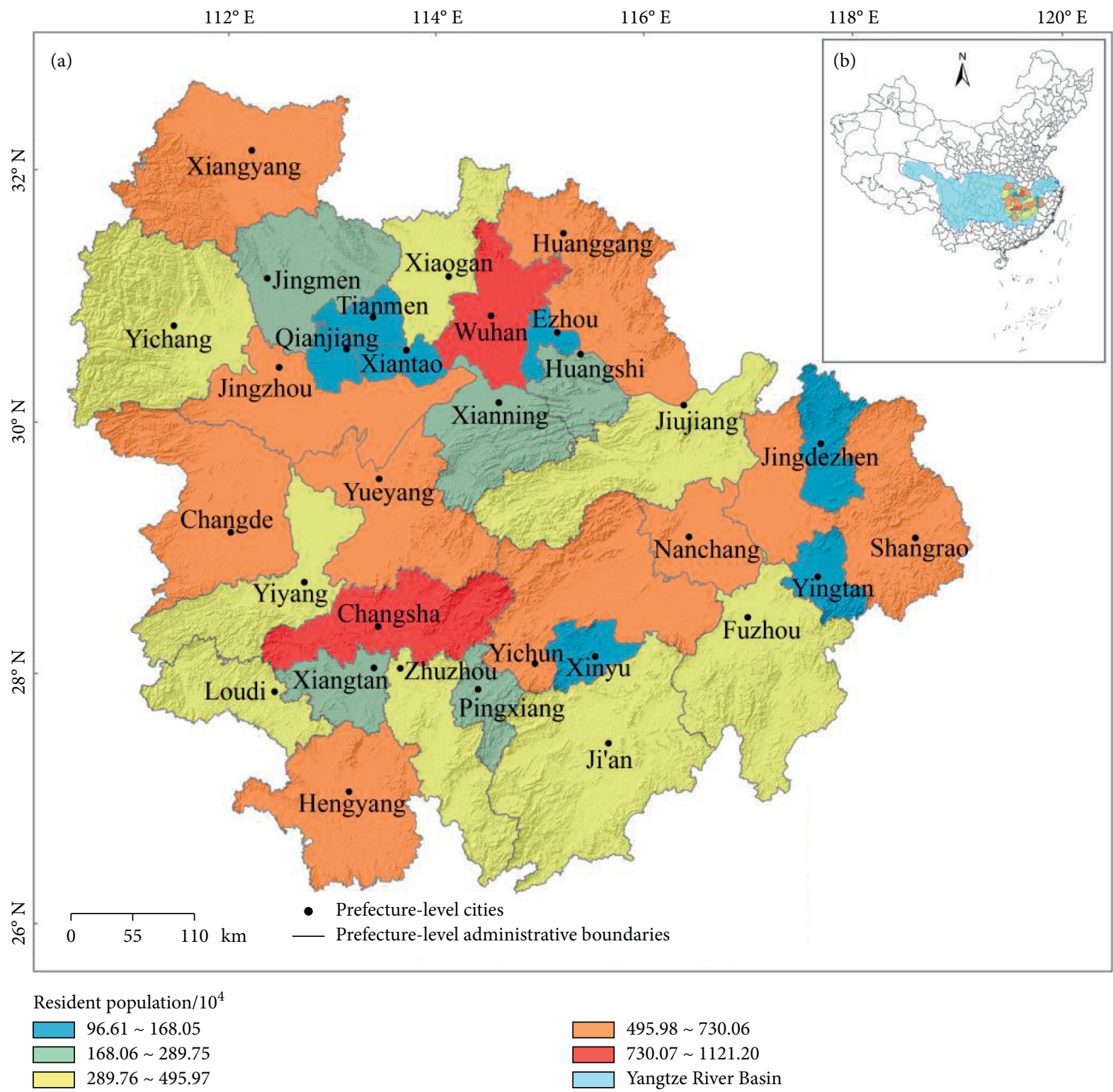

Figure 1: Study area: (a) spatial distribution of resident population; (b) the position of the study area in the Yangtze River Basin and China.

through gathering the Autonavi migration index during the Spring Festival travel rush in 2019, 2020, and 2021, the intercity population migration networks within the urban agglomeration are constructed to analyze spatiotemporal characteristics and resilience changes. The data analyzed in this study, Autonavi migration index, were derived from traffic big data provided by the Autonavi Company, one of the solution providers of digital map, navigation, and location service in China. According to statistics from the 2021 China Mobile Internet Development Report, as of the end of 2020, the number of monthly active users of Amap reached 555.86 million, while the Baidu map ranked second with 434.73 million users (https://www.questmobile.com.cn/ research/report-new/143). That is, the Amap service ranked first among the travel service apps (Baidu Map, Didi Chuxing, and Tencent Map). Autonavi migration data, similar to Tencent migration and Baidu migration data, represents the index of population migration between cities, which is obtained by the number of migrants in each city and the total number of migrants in China. Each record of Autonavi migration data is mainly composed of four fields: source city, target city, actual migration index, and migration willingness index. The actual migration index is the record after completing the travel purpose from one city to another via Autonavi navigation service in Amap, which, to a large extent, represents the population travel on the highway. The migration willingness index refers to the behavior of searching target places and then planning driving routes via Autonavi navigation, that is, people's travel desire, rather than the real migration behavior. Through screening and cleaning the data for 120 days during the SFTR in 2019 (from January 21 to March 1, 2019), 2020 (from January 10 to February 18, 2020), and 2021 (from January 28 to March $8,2021)$, the available data of three periods are obtained. 


\subsection{Methods}

3.2.1. Construction of Population Flow Network. According to the complex network theory, regarding cities and connection between cities as nodes and edges, respectively, the population flow network among 31 cities was described. A directional weighted matrix $L=\left(L_{i j}\right)$ was used to characterize the flow of the population during one day, where the $L_{i j}$ is the population flow intensity from city $i$ to city $j$. Then, $40 \times 3$ directed weighted asymmetric matrices of $31 \times 31$ were constructed based on Autonavi migration data. The matric can be expressed as follows [3]:

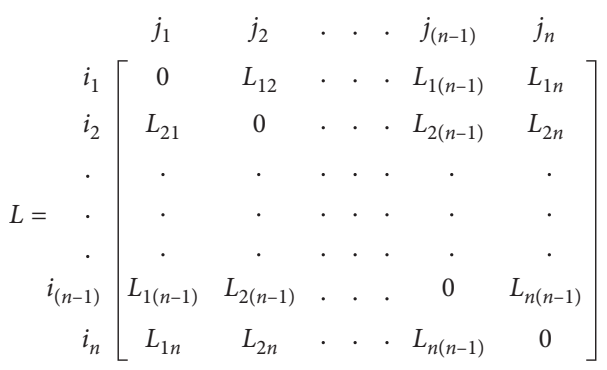

\subsubsection{Social Network Analysis Methods}

(1) Weighted degree. The weighted degree represents the sum of the weights of the arcs connected with a city node, the larger the value, the stronger the connection between cities. In a directional weighted network, the weighted degree of a node is the sum of the weighted out-degree and the weighted in-degree of the one. Weighted out-degree measures the total population flow intensity from city node $i$, while weighted in-degree measures the total population flow intensity to node $i$. The formula is as follows:

$$
W_{i}=W_{i}^{\text {in }}+W_{i}^{\text {out }},
$$

where $W_{i}$ is the weighted degree of city $i, W_{i}^{\text {in }}$ is the weighted in-degree of city $i$, and $W_{i}^{\text {out }}$ is the weighted out-degree of city $i$.

(2) Node symmetry. Node symmetry is used to describe the difference reflected by the in-degree and out-degree of every node [27]. However, the number relationship of intercity migration cannot be well reflected only by the in-degree and outdegree. Therefore, this study introduced the population flow intensity of the directed weighted network to calculate the node symmetry index (NSI) and then judge whether the city node is the receiver or the sender. The formula of NSI of the city node $i$ is as follows:

$$
\mathrm{NSI}_{i}=\frac{W_{i}^{\mathrm{in}}-W_{i}^{\text {out }}}{W_{i}^{\text {in }}+W_{i}^{\text {out }}}
$$

(3) Clustering coefficient. The clustering coefficient is used to describe the interconnection level of nodes [28]. When certain nodes are closely connected, they can form a network cluster. The formula of clustering coefficient is as follows [25]:

$$
C_{i}=\frac{2 B_{i}}{m_{i}\left(m_{i}-1\right)},
$$

where $C_{i}$ is the clustering coefficient and $B_{i}$ is the number of paths between the node and the neighboring nodes of $m_{i}$.

(4) PageRank algorithm. PageRank algorithm is originally used to evaluate the rank of a web page through the complicated hyperlink relationships in a network [29], revealing the importance of a particular web page relative to other web pages in the network. It is used herein to measure the criticality of city nodes in the population flow network. The calculation equation of the PR value of node $i$ is as follows [30]:

$$
\operatorname{PR}\left(p_{i}\right)=\frac{1-d}{N}+d \sum_{p_{j} \in M(i)} \frac{\operatorname{PR}\left(p_{j}\right)}{L(j)},
$$

where $N$ is the number of all nodes, $d$ is the damping coefficient (a value between zero and one), $M(i)$ is the set of nodes connected to node $i$ and out of the chain (in-degree), and $L(j)$ is the number of nodes connected by node $j$ to the outside (out-degree).

\subsubsection{Assessment of Network Structure Resilience.} Network structure resilience refers to the ability of the network system to respond to sudden external shocks and disturbances. For the urban network system, the interference to a certain urban node may lead to the domino effect, thereby affecting the partial cascading of the network [31]. The resilience of the network structure herein is measured by the response of network after node failure and node attack. Node failure mainly considers the impact of natural disasters on different urban nodes, such as mud-rock flow, snowstorm, haze, etc. The resilience of the urban network structure in the face of interference is observed via removing the city nodes in turn. Node attack mainly considers the impact of man-made damages, such as terrorist attacks, military conflicts, or other artificial forces, remove nodes in the descending rank of node criticality (PR value), simulating the nodes fail from the most "important" ones [17]. Both strategies assume that the node will fail immediately after removal, and all edges are directly connected to the node will also be removed.

Network efficiency is a physical quantity used to describe the diffusion ability of elements among the networks, which is generally evaluated by path-related indicators of the network. In a network with high network efficiency, the propagation and exchange of elements between nodes can be realized more quickly, which is conducive to promoting the 
learning, innovation, and communication among nodes, and can enhance the resistance of the network to external shocks so that the network has a higher resilience. The network efficiency $(E)$ has been widely regarded as an important metric to measure the network response $[32,33]$; the larger the value, the better the performance of the network. The changes to its value indicate changes in global efficiency due to disturbances. For the directional weighted network, the formula is as follows:

$$
E=\frac{1}{N(N-1)} \sum_{i \neq j \in G} \frac{1}{D_{i j}} \times \mathrm{AW}_{i j}
$$

where $D_{i j}$ is the shortest path length from urban node $i$ to $j, N$ is the number of urban nodes in the network, and $G$ is the set of urban nodes in the network after removing a certain node, $\mathrm{AW}_{i j}$ is the average weighted degree of the edges in the shortest path from node $i$ to node $j$.

\section{Results}

4.1. Characteristics of Population Migration during SFTR. To analyze the characteristics of population migration of urban agglomeration in the middle reaches of Yangtze River during SFTR, a time-sequential distribution chart of the daily summary (average) migration scale during the SFTR in 2019, 2020, and 2021 was produced (Figure 2). Figure 2 shows the following characteristics of the population flow. First, in a year, the changing trend of migration willingness and actual migration scale is basically consistent during the study period, and the migration willingness scale is always higher than the actual migration scale. Second, the migration willingness and actual migration index before the Spring Festival (the 16th day) shows a trend of first increase and then decrease. Due to the influence of the prevention and control policies of COVID-19, the people migration scale in 2020 and 2019 is higher than that in 2021. In addition, compared with 2019, the peak of population migration before the Spring Festival in 2020 and 2021 is slightly delayed. Third, due to the particularity of COVID-19, to prevent COVID-19 from rapidly spreading with population movements, the Wuhan Municipal Government completely closed the city at 10:00 a.m. on January 23, 2020 (the 14th day). Nevertheless, approximately 5 million people still left Wuhan [34]. Therefore, the migration willingness and actual migration index after the Spring Festival (the 16th day) in 2020 show a downward trend, which is far lower than the migration level in the same period in 2019 and 2021. With the effective prevention and control of COVID-19, the population migration after the Spring Festival in 2021 shows a rapid increasing trend compared to that before the year. On the last day of the Spring Festival Holiday (the 21st day), the population migration scale of the study area in 2021 exceeds that in 2019, and reaches the largest scale of people migration during SFTR. As the last traditional festival during SFTR, the Lantern Festival also ushered in a small peak of population flow. Since then, the population transportation of SFTR has gradually come to an end.

\subsection{Spatiotemporal Characteristics of Population Flow Networks}

4.2.1. Spatiotemporal Patterns of Popular Flow Networks. With the help of ArcGIS software, the population flow networks were visualized based on the summarized directional weighted matrix of population flow intensity. The overall spatiotemporal pattern of population flow networks of urban agglomeration during the SFTR in the middle reaches of Yangtze River is presented in Figure 3. Meanwhile, the population migration intensity is divided into four levels, the higher the level, the stronger the population migration scale between cities.

Figure 3(a) shows the spatial pattern of the population flow network in 2019. From the results of the grading map, population flow between cities in the first level (81-177) mainly revolves around 12 routes around Wuhan, Changsha, and Nanchang, showing a characteristic of large-scale and basically symmetrical population migration between neighboring cities. The spatial pattern of the second level (41-80) still presents a central radial structure, which to some extent is a complement of the surrounding cities of the first level. Only the Changsha-Zhuzhou-Xiangtan city group shows the population flow across city space. In addition, it is found that there is a relatively strong connection between Xiangtan and Zhuzhou, and no major core cities were involved. The third level (10-40) mainly reflects the interaction among cities around the core cities, characterized by more in the northwest and less in the southeast. The fourth level $(<10)$ accounts for about $84 \%$ of all routes, indicating that the intensity of population flow across region space in most cities is still weak.

The spatial pattern of the population flow network in 2020 (Figure 3(b)) indicates that the population flow routes of the first three levels are extremely sparse. The number of routes is 3,12 , and 68 , respectively. The routes in the first level are only Wuhan-Xiaogan, Xiangtan-Changsha, and Changsha-Yiyang. As the core city of the Poyang Lake city group, Nanchang only appears in the second level, and the connection with Yiyang is relatively weak. The rest of the population flow connections also correspond to the first level in 2019. It indicates that the outbreak of COVID-19 in Wuhan had a serious impact not only on Wuhan and its surrounding cities but also on the closely linked cities within the urban agglomeration. Although there are population flow routes across region space in the third level, the number is few. For instance, Huanggang-Jiujiang, Jingzhou-Yueyang, and Changde-Jingzhou. The population flow routes in the fourth level have a relatively large scale, accounting for about $90 \%$ of all routes. This illustrates that during the epidemic in Wuhan, the closure policy had a greater impact on all cities within the urban agglomeration, causing an attenuation on the Spring Festival travel rush in China.

The population flow network during the SFTR in 2021 is shown in Figure 3(c) with levels 1 to 3 including 16, 23, and 103 routes, respectively. Compared with that of 2019, its population migration intensity has increased; on the other hand, both Wuhan and Nanchang are joined by new urban 


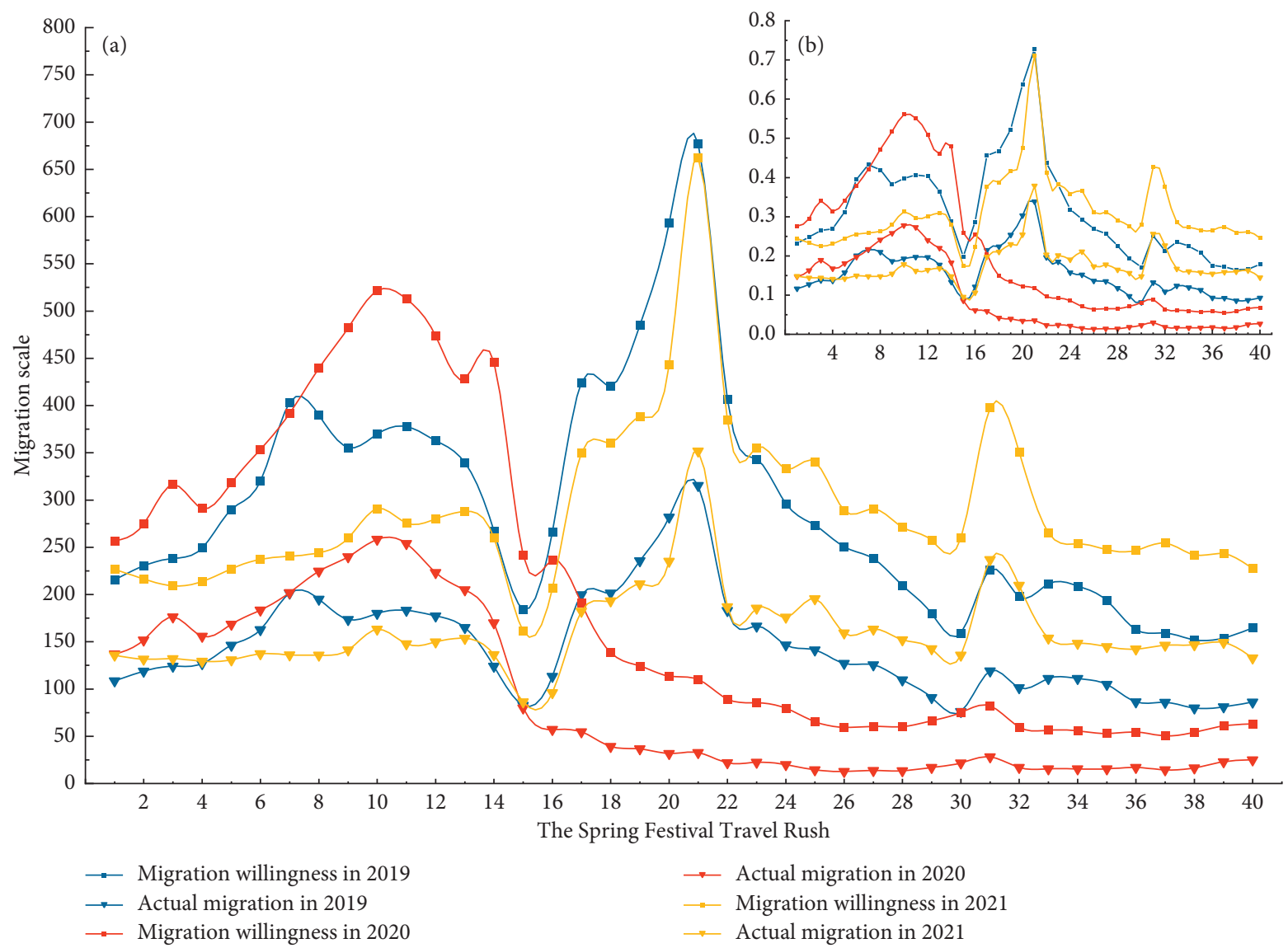

FIGURE 2: The population migration scale of the study area during the SFTR: (a) daily summary migration scale; (b) daily average migration scale. Blue, red, and yellow segments represent 2019, 2020, and 2021, respectively. The box and inverted triangle represent the migration willingness and the actual migration scale, respectively.

nodes at the first level, such as Ezhou and Jiujiang. In the second level, the population flow routes around Poyang Lake city group have increased significantly, which are reflected in Yingtan and Fuzhou. There are no significant differences in the third level of the population flow networks in 2019 and 2021. Surprisingly, the number of the routes in the fourth level is almost the same as in 2019 and 2021.

From the perspective of the urban agglomeration in the middle reaches of the Yangtze River, there are 930 routes in each population flow network during the three periods, that is, the population flow networks are all strongly connected graphs. However, the intensity of population flow during the SFTR in 2019, 2020, and 2021 is quite different. The maximum migration scale of the population flow network during the SFTR from 2019 to 2021 is 177,87 , and 213, respectively. Correspondingly, the average migration scale of each route is $6.18,3.68$, and 6.90 , respectively. In addition, the spatial distribution of population flow during the SFTR in the urban agglomeration also shows temporal and spatial differences. In general, the networks present a spatial distribution pattern of "dense on the northwestern side and sparse on the southeastern side". Polycentricity is a prominent feature of the region. Specifically, the population flow between core cities (Wuhan, Changsha, and Nanchang) is relatively weak. In the first and second levels, no corridors connecting the three suburban groups are formed. The population migration intensity between Wuhan and Changsha during the SFTR in 2019 and 2021 is only at the third level. What's worse, its connection is weaker in 2020, only at the fourth level. Second, the population flow connections are mainly gathered on the northwestern side, between the Wuhan metropolitan area and the Changsha-Zhuzhou-Xiangtan city group, which is the main driving force for the development of the region. Only in 2020, due to the quarantine of Wuhan, the connection between them tends to weaken. In comparison, the interaction between the Poyang Lake city group on the southeast side and other regions is weaker, especially in 2020. Nevertheless, the results of their urban cluster structure reveal that the population flow networks of three periods surprisingly have the same urban cluster structure. That is, 13 cities in the Hubei province form cluster 0,8 cities in the Hunan province form cluster 1 , and 10 cities in the Jiangxi province form cluster 2 . The cluster structure in different periods is completely consistent with the provincial boundary, indicating that there is an obvious provincial boundary segmentation effect on the urban agglomeration, which makes the population flow elements within the urban agglomeration focus only on the interior of provinces, and the integration level of the region is relatively low. 

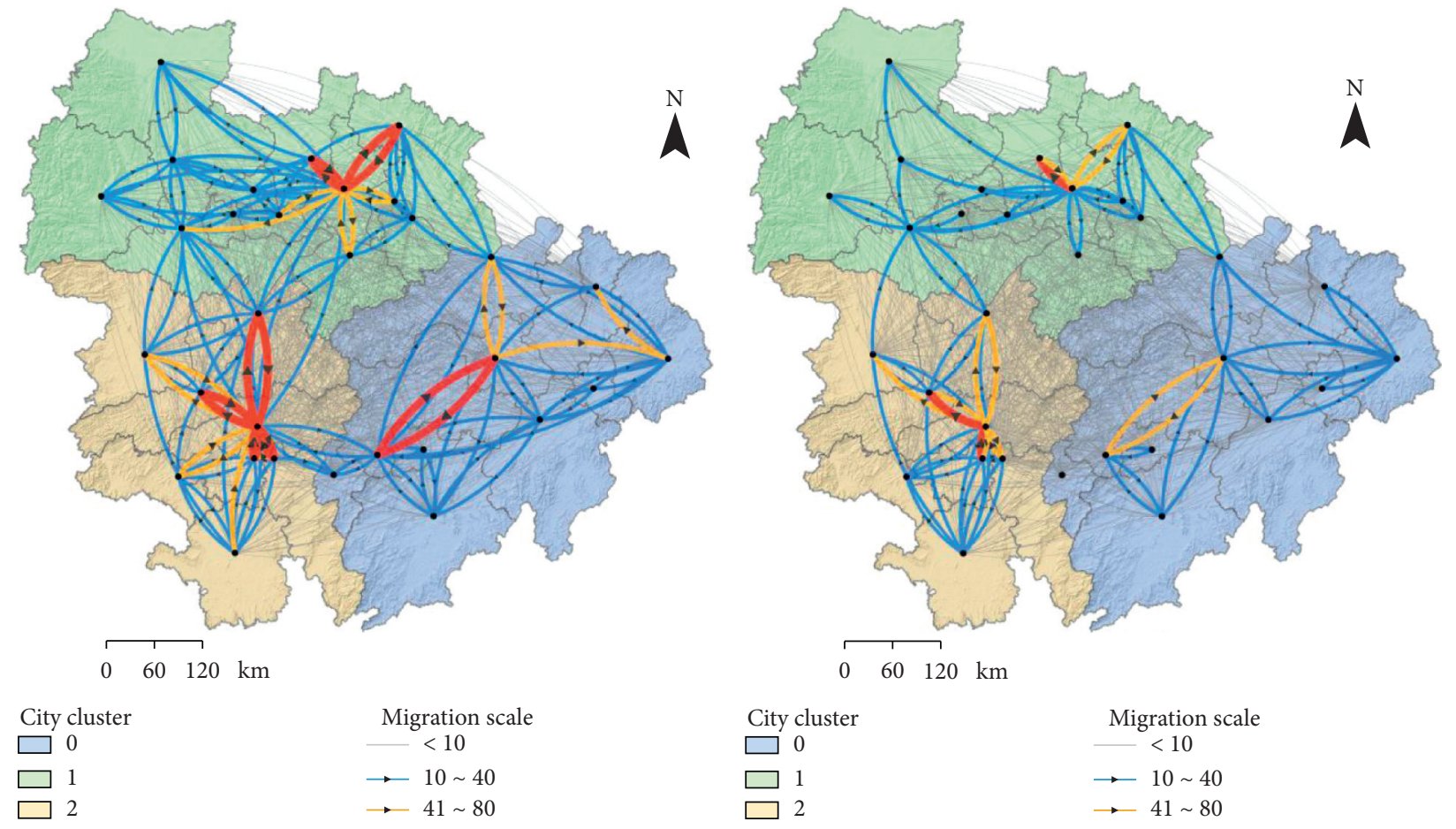

$$
\begin{aligned}
& \text { Migration scale } \\
&<<10 \\
& \rightarrow-10 \sim 40 \\
& \rightarrow \quad 41 \sim 80 \\
& \rightarrow \quad 81 \sim 177
\end{aligned}
$$

(a)

$$
\begin{gathered}
\text { Migration scale } \\
<10 \\
\rightarrow \quad 10 \sim 40 \\
\rightarrow \quad 41 \sim 80
\end{gathered}
$$

(b)
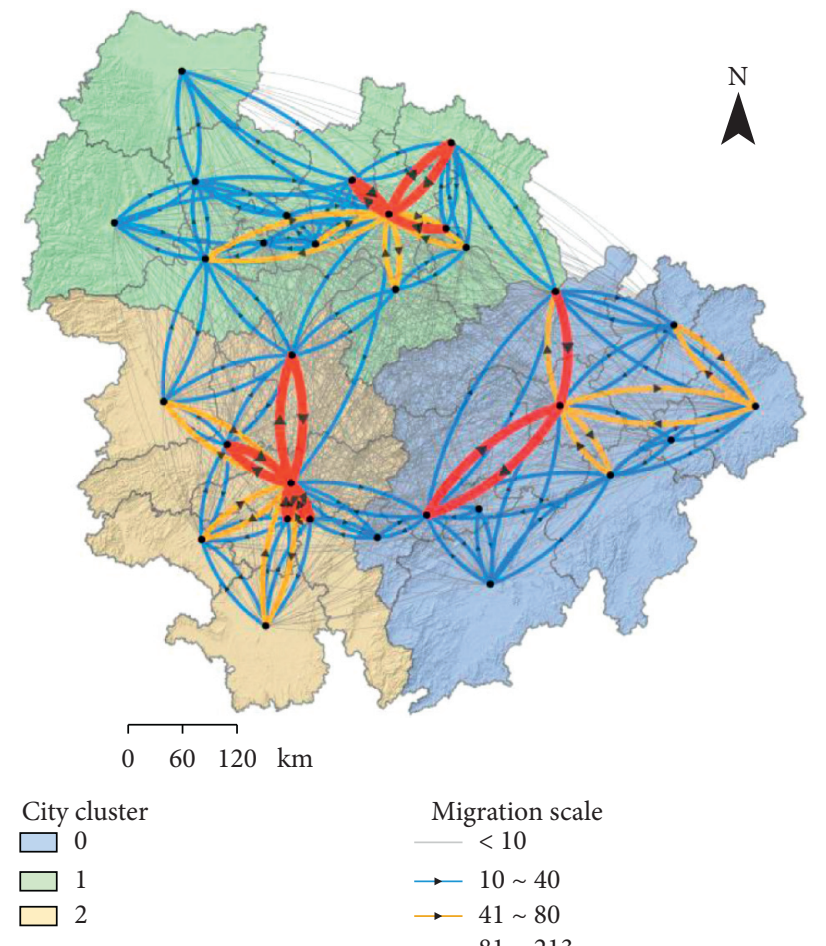

$$
\begin{gathered}
\text { Migration scale } \\
<10 \\
\rightarrow-10 \sim 40 \\
\rightarrow \quad 41 \sim 80 \\
\rightarrow \quad 81 \sim 213
\end{gathered}
$$

(c)

FIgURE 3: Population flow networks of the middle reaches of Yangtze river: (a) 2019; (b) 2020; and (c) 2021. The color of the route represents the intensity of population flow, and the change from gray to red represents an increase in the intensity. The color of the polygon represents city cluster. 
4.2.2. Popular Migration Routes of the Study Period. By drawing the actual migration index and the migration willingness index, the popular migration routes (Figure 4) during the three periods of SFTR were obtained. The popular migration routes in 2019 are Xiaogan-Wuhan, Wuhan-Xiaogan, Xiangtan-Changsha, Huanggang-Wuhan, Wuhan-Huanggang, Yiyang-Changsha, and Changsha-Xiangtan. In 2020, the routes are XiangtanChangsha, Wuhan-Xiaogan, Changsha-Yiyang, Changsha-Yueyang, Xiaogan-Wuhan, Changsha-Xiangtan, and Wuhan-Huanggang. In 2021, the routes are Xiaogan-Wuhan, Xiangtan-Changsha, Wuhan-Xiaogan, Yiyang-Changsha, Wuhan-Huanggang, Huanggang-Wuhan, and Changsha-Xiangtan.

Combined with the popular flow networks (Figure 3), we can see clearly that the most popular migration routes in 2019 mainly focus on Wuhan, involving the nearest cities Xiaogan and Huanggang. Then, the routes are Xiangtan and Yiyang to Changsha. They reflect the migration effect from surrounding cities to core cities. During the SFTR in 2020, under the influence of COVID-19, the popular migration routes in the study area have changed greatly compared with that in 2019. As a subcore city, Changsha has become the dominant city of population migration. In addition, it is characterized by the popular migration from the core city to the surrounding cities. Popular migration routes in 2021 are similar to those in 2019, mainly centered around the two core cities of Wuhan and Changsha, and the difference is mainly reflected that the population migration intensity in 2021 is higher than that in 2019.

4.2.3. Dominant Flow and Node Symmetry. By extracting the dominant flow (maximum inflow or outflow) data of each city in the urban agglomeration, the population flow paths of the dominant flow during the period of SFTR were drawn. The results are expressed in the form of curves, whose colors represent different city clusters (Figure 5). In addition, in order to analyze the characteristics of urban population inflow and outflow more concretely and intuitively, we overlay the results of the node symmetry index with the dominant flow. According to the results of the node symmetry index, the city nodes were divided into four types, such as strong outflow, weak outflow, weak inflow, and strong inflow nodes.

From the dominant flow and city clusters, the number of city clusters of population dominant flow in the SFTR periods of 2019, 2020, and 2021 in the study area is 4, 5, and 3, respectively. There are different structures of city clusters corresponding to each period, among which 2019 and 2021 are more similar, showing a stronger clustering degree of 2021 than that of 2019. Specifically reflected as follows: for cluster 0,13 cities in Hubei province not only have the same clustering characteristics but also have the same direction of population dominant flow in 2019 and 2021. However, the cluster was divided into three smaller city clusters (clusters 0 , 3 , and 4) in 2020 (Figure 5(b)). In addition, Jingmen, Jingzhou, Xiangyang, and Tianmen, which are used to taking Wuhan as the first inflow city, changed their inflow targets to the adjacent marginal cities. For cluster 1, the structure of population dominant flow in 2019 and 2021 is the same. Pingxiang, which belongs to cluster 2 in 2019 and 2021, had been attracted to cluster 1 with Changsha as the core in 2020 . Besides, the dominant flow city in Changsha changed from Xiangtan to Yiyang. For cluster 2, although the three periods have different characteristics, on the whole, the changing trend is from weak to strong. In 2019, cluster 2 has not yet been merged with cluster 3 on the edge of the urban agglomeration. However, as the dominant flow city in Shangrao changed from Jingdezhen to Nanchang and the dominant flow city in Pingxiang changed from Changsha to Yichun, 10 cities in the Jiangxi Province (city groups around Poyang Lake) within the urban agglomeration formed a larger city cluster. Therefore, there were only three urban clusters in 2021, and they were relatively complete boundaries divided by provincial regions.

Node symmetry describes the difference between the population inflow and outflow interactions for every city node. The outflow nodes are the nodes with a negative node symmetry index. They are more important as senders in the population flow network. According to the negative value, they were divided into two types: strong outflow and weak outflow nodes. Correspondingly, the inflow nodes, including strong inflow and weak inflow nodes whose node symmetry index is positive, are primarily receivers in the network.

From the perspective of the node types (Figure 5), the strong outflow nodes mainly distribute around the core cities (Wuhan, Changsha, and Nanchang). The spatial distribution of the three periods is slightly different. As far as Wuhan metropolitan area is concerned, there are only two strong outflow cities (Xiantao and Ezhou) in 2019, all weak outflow and inflow cities in 2020, and three strong outflow cities (Xiantao, Ezhou, and Xiaogan) in 2021. Consequently, Wuhan has changed from a weak inflow node in 2019 to a strong inflow node in 2021. As a strong inflow city in 2019 and 2021, Changsha changed its direction and became a weak inflow node in 2020. Although Nanchang is known for its weak inflow, it turned around in 2020 and became a weak outflow node. Some weak outflow cities in 2019, such as Jingmen, Xianning, Yueyang, and Loudi, became weak inflow nodes in 2020, but quickly returned to the state of 2019 in 2021. In addition, there are some from weak to strong outflow cities such as Xiaogan, Qianjiang, and Yiyang, as well as inflow cities such as Wuhan and Pingxiang.

To sum up, the population flow of most urban nodes presents asymmetric characteristics during the SFTR in the study area. The core cities gradually show the characteristics of inflow from weak to strong, while its neighboring cities show the phenomenon of a strong outflow. This indicates that during the period of SFTR, the provincial capital cities still play a powerful central collection role. They are not only the travel targets of the surrounding cities, but also the destinations of the marginal cities.

4.3. Node Criticality. To estimate the importance of city nodes, on the premise of considering the intensity of population flow, the PageRank algorithm was applied to 


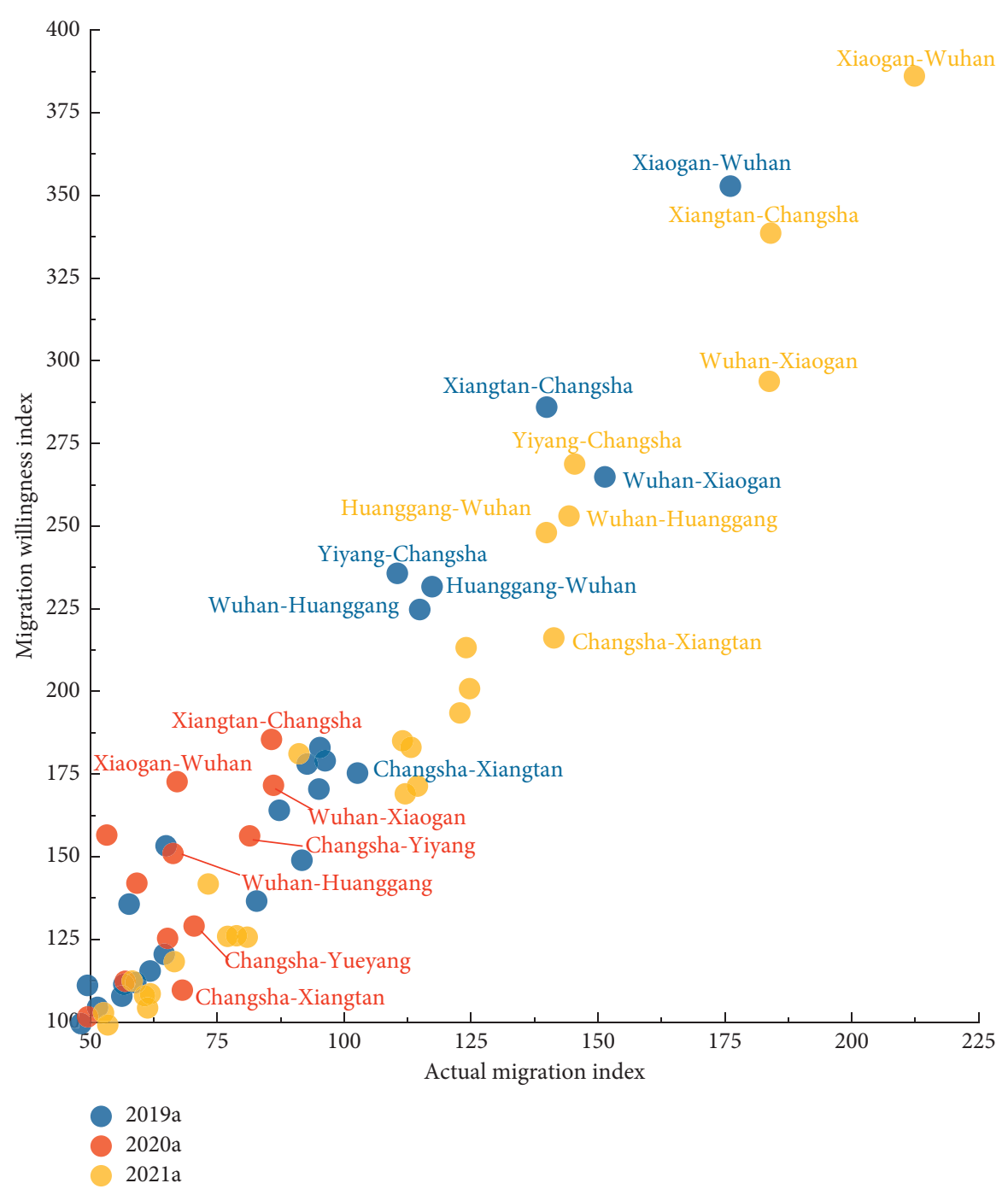

Figure 4: Popular migration routes in 2019, 2020, and 2021. The blue, red, and yellow represent the routes in 2019, 2020, and 2021, respectively.

calculate the PR values of cities in the population flow networks, with the damping coefficient set to 0.85 . The higher the PR of a city, the higher status in the network and the more critical the city node is, accordingly. The PR values of city nodes during the SFTR in the study area were divided into five different levels via Natural Breaks, which can be shown in Figure 6. The nodes with higher PR values are mainly concentrated in the provincial capital cities. Although Nanchang jumped to the first level in 2021 because of its higher PR value (0.63), it still lags far behind Wuhan (0.11) and Changsha (0.10), which reveals that Nanchang, the core leading city of the Poyang Lake urban group, is emerging and is expected to form a tripartite confrontation with Wuhan and Changsha in the urban agglomeration. Jingzhou, as a city with a strong inflow of population in 2019 and 2020, has its status improved significantly in 2020. Six cities in its surroundings also rose in statuses, such as the three cities Changde, Yiyang, and Yueyang, from the third to the second level, while Xiangyang, Jingmen, and Yichang rose from the fourth to the third level. In the normal years of
2019 and 2021, the cities with a second-level and third-level PR value are mainly distributed around and between capital cities, which are bridges for the communication of population elements between capital cities. The cities with a low PR value are some marginal cities, such as Fuzhou, Jian, Hengyang, Loudi, Changde, Yichang, etc. For the cities with a lower PR value, such as Pingxiang, Xinyu, Yingtan, Tianmen, Qianjiang, etc., due to the small number of permanent residents, they have no advantages in the population flow network, thereby having the lowest status of cities.

\subsection{Resilience Analysis of Urban Network Structure}

4.4.1. Network Response to Node Failure. As a kind of network disruption, the failure of an urban node may be the loss of city caused by disaster; for example, Wuhan was quarantined from January 23 to April 8, 2020 because of the person-to-person transmission characteristic of COVID-19. To reflect the impact of disasters that different urban nodes 

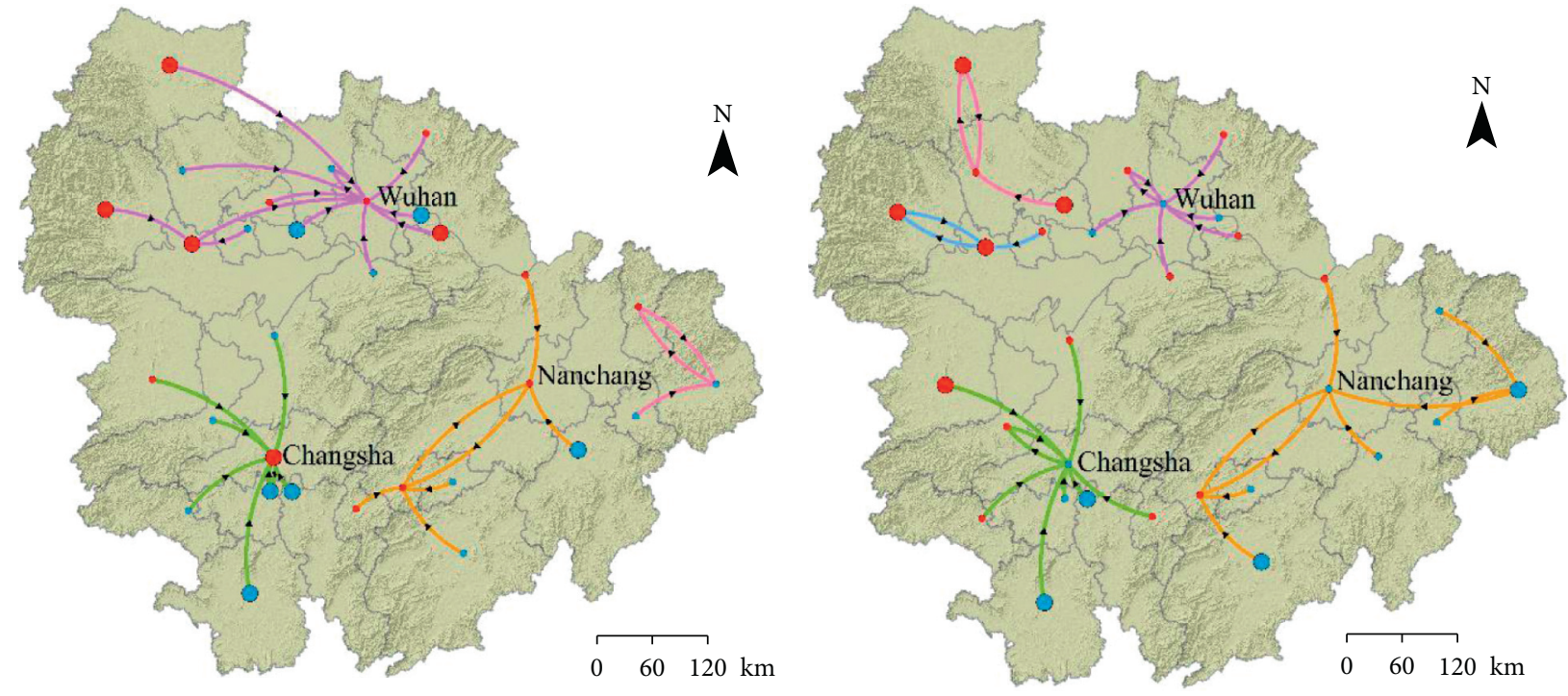

City cluster
$\rightarrow 0$
$\rightarrow-1$
$\rightarrow-2$
$\rightarrow-3$

City node type

O Strong outflow

- Weak outflow

- Weak inflow

- Strong inflow

(a)

$$
\begin{aligned}
& \text { City cluster } \\
& \rightarrow-0 \\
& \rightarrow-1 \\
& \rightarrow \quad 2 \\
& \rightarrow \quad 3 \\
& \rightarrow \quad 4
\end{aligned}
$$

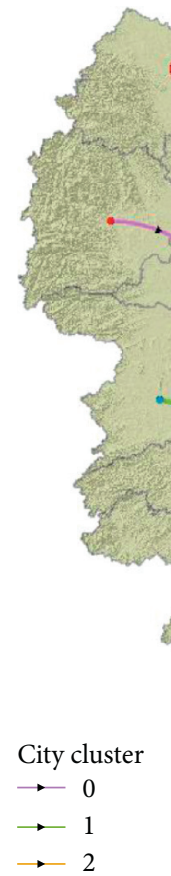

(b)
City node type
O Strong outflow
- Weak outflow
- Weak inflow
- Strong inflow 


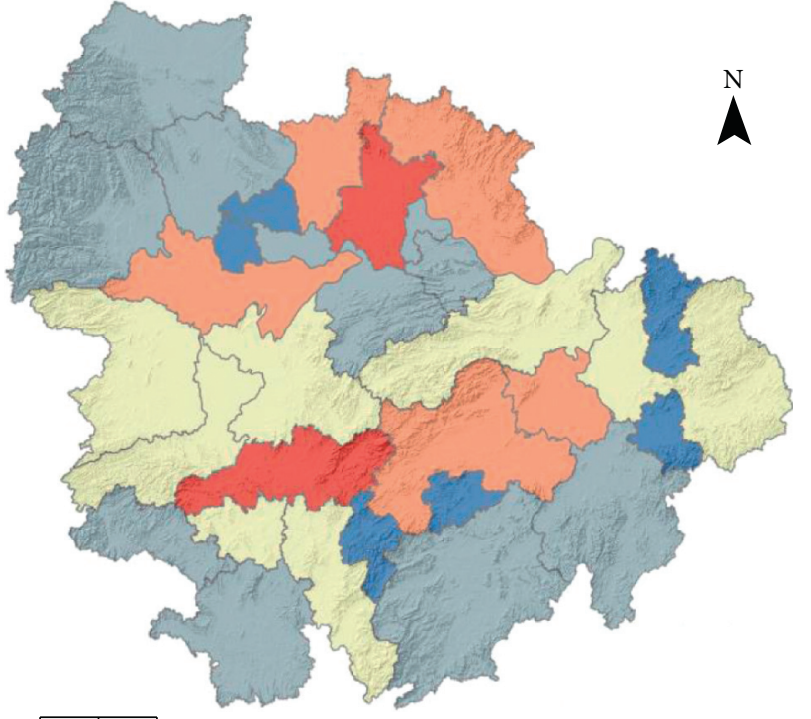

$0 \quad 60 \quad 120 \mathrm{~km}$

PageRank value

$\square 0.013 \sim 0.017$
$\square 0.018 \sim 0.025$
$\square 0.026 \sim 0.038$

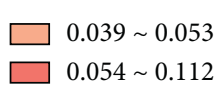

(a)

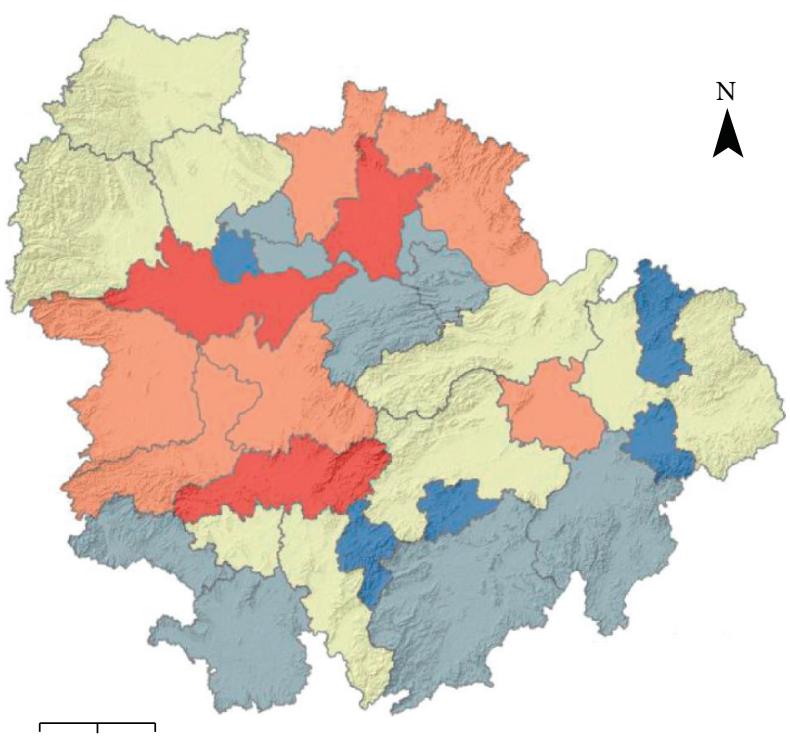

$\begin{array}{llll}0 & 60 & 120 & \mathrm{~km}\end{array}$

PageRank value

$\square .011 \sim 0.017$
$\square \quad 0.018 \sim 0.025$
$\square \quad 0.026 \sim 0.038$

$0.039 \sim 0.053$

$0.054 \sim 0.099$

(b)

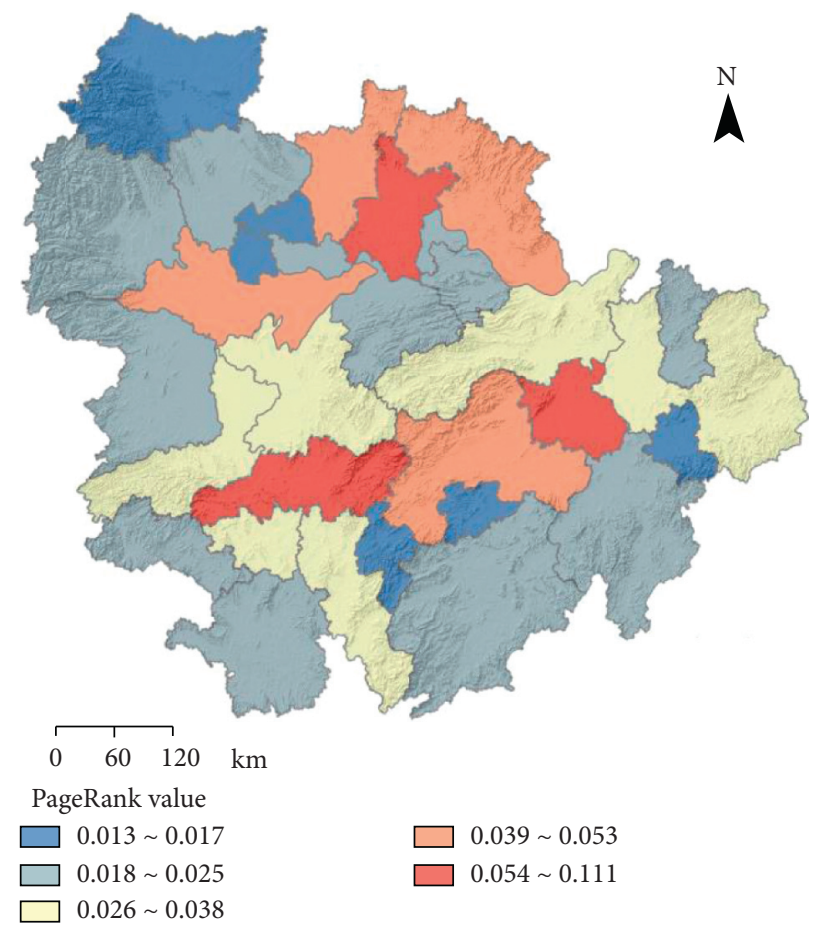

(c)

Figure 6: Spatial distribution of the PageRank value: (a) 2019; (b) 2020, and (c) 2021. The color from blue to red represents the different levels from low to high of PageRank.

structural resilience of the population flow networks during the SFTR in the study area. From the overall network efficiency after urban node failures, the result of 2021 (11.05-14.50) is slightly higher than that in 2019
(10.27-13.00), far higher than that in 2020 (6.28-8.24), revealing that after a severe epidemic, the propagation efficiency of the population flow networks in 2021 has been improved, and has exceeded that in 2019. However, in 2020, 


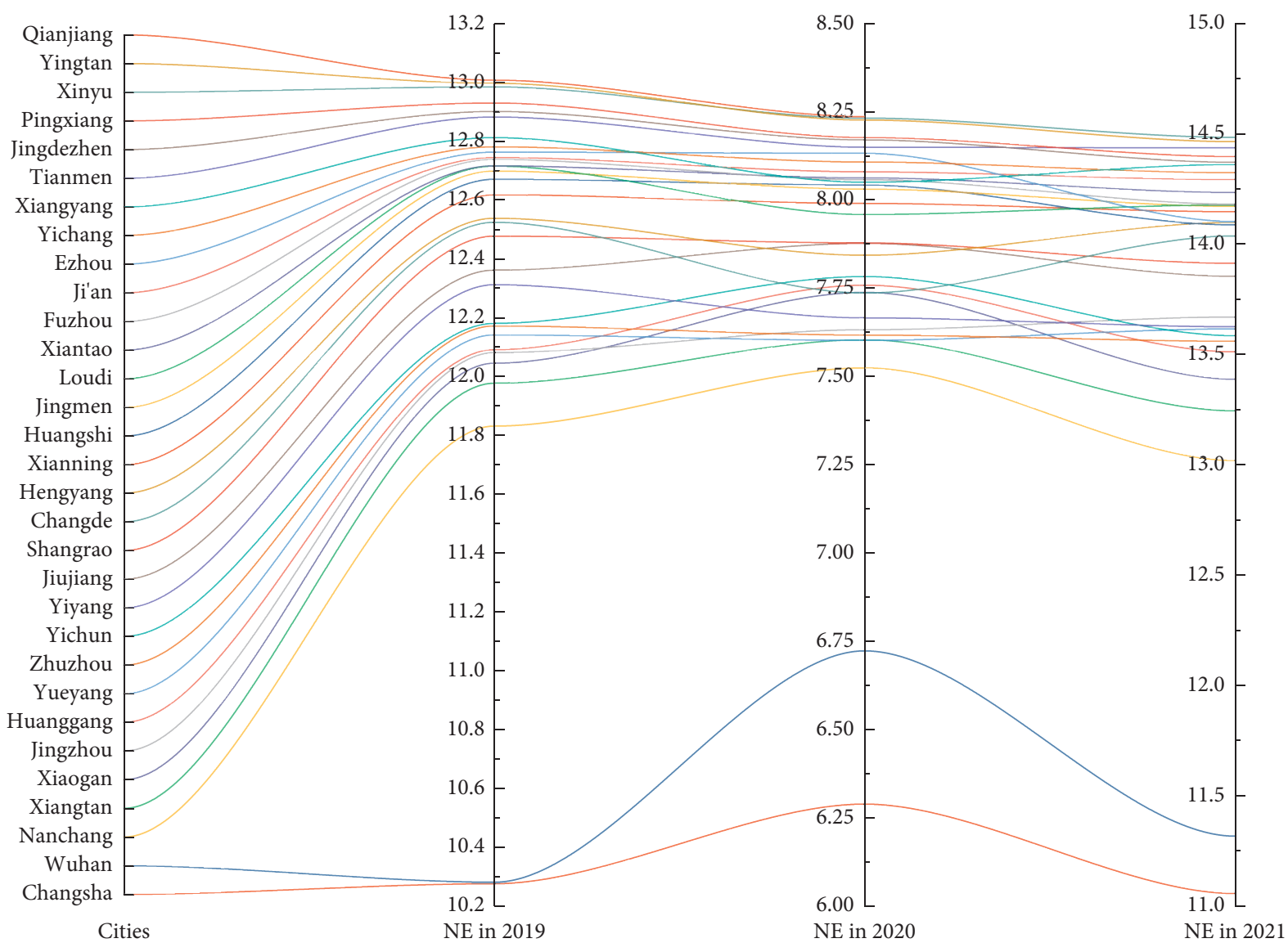

Figure 7: The changes in the network efficiency (NE) after node failure in different cities in 2019, 2020, and 2021.

the transmission efficiency of the population flow network is at a low point, which reflects the fragility of the network structure.

It is worth noting that after the failures in Changsha or Wuhan in 2019, the response of population transportation networks is almost the same, illustrating that the two primary core hub cities in the study area have relatively equal contributions to the Spring Festival travel rush. Obviously, because of the disturbance of Wuhan and travel restrictions in 2020, the overall network efficiency has reduced. Changsha has become the leading city of the urban agglomeration and has taken on the responsibility of population transportation. In addition, the response of the network to Xiaogan or Huanggang failures is insensitive because of urgent travel constraints caused by the rising number of patients with infection, while Zhuzhou, Yueyang, Yiyang, Changde, Hengyang, and Loudi within the Changsha-Zhuzhou-Xiangtan city group have a significant increase in the impact on the resilience of the network structure, because they are far away from the areas seriously affected by COVID-19. Nanchang, however, as the third core city of the study area, as well as the third city that affects the resilience of network structure in each period, still has not been prominent in the overall network. In other words, during the SFTR in 2020, the Wuhan metropolitan area has a serious disturbance on the population flow, which made the population flow barycenter in the urban agglomeration more inclined to the Changsha-Zhuzhou-Xiangtan city group, thereby increasing the pressure of epidemic prevention and control in the Hunan province. However, because of its lower level in the population flow network, the Poyang Lake city group does not play an obvious role in population diversion, thus the pressure of epidemic prevention and control is relatively small.

The first five cities in 2021 that affect the structural resilience of the population flow network are consistent with those in 2019, namely, Changsha, Wuhan, Nanchang, Xiangtan, and Xiaogan. Moreover, the impact of failures of the four cities, Zhuzhou, Yichun, Huangshi, and Ezhou, on the resilience of the network structure has been significantly enhanced due to their strong interaction with the core cities in the subregion. On the contrary, there are some cities whose population collection and distribution capacity is significantly reduced, such as Jingzhou, Xianning, and Xiantao, which might be subjected to homogeneous radiation from the surrounding cities. In addition, there are always some marginal cities, such as Qianjiang, Yingtan, Xinyu, Pingxiang, Jingdezhen, Tianmen, etc. While their risk of failure may not cause irreparable damage to the urban agglomeration, alienated relationships with other cities in the region may lead to their inability to obtain rapid resources. Therefore, attention should be paid to the 
improvement of the marginal nodes, which will not only help to enhance regional resilience but will also be of great benefit to the ability of the city itself to resist risks and disasters.

4.4.2. Network Response after Node Attack. As another kind of network disruption, node attack usually attacks the node with the maximum load, such as maximum degree, weighted degree, or centrality. For regional urban systems, malicious attacks may be terrorist attacks, military conflict, or other human forces, which are unpredictable and uncontrollable. Therefore, an attack against a certain city may lead to a wide range of network topology failures, thereby leading to the loss of urban network functions. According to the descending order of node criticality, the attacks on the city nodes were simulated. It is assumed that all links connected to a node will be removed after it was attacked, and the routes among other nodes will be redistributed. Figure 8 is the urban network response to node attacks during the three periods of SFTR. The $x$-axis is the percentage of nodes attacked, and the $y$-axis is the corresponding network efficiency. We can see that as the percentage of failed nodes increases, the resilience of the network structure decreases continuously. Obviously, although there is the highest population flow intensity of urban agglomeration in the middle reaches of the Yangtze River during the SFTR in 2021, its descent rate of the network efficiency is the fastest. Especially when attacking Nanchang and Jingzhou, the cities ranked third and seventh, and the network performance will be lower than that of 2019. Secondly, when the percentage of urban nodes that failed reaches $22 \%$, the response of the population flow network in 2019 is almost the same as that in 2021, which is about 5.80. After that, with the interruption of urban nodes, the network efficiency in 2021 still declined at the fastest speed, and the resilience of urban network structure is always lower than that that in 2019. When the attack rate of urban nodes is slightly higher than $93 \%$, the network efficiency almost drops to 0 , which is similar to that in 2020. However, when the urban network efficiency drops to 0 in 2019, the attack rate of nodes exceeds $96 \%$. This indicates that the urban population flow network of the study area during the SFTR in 2019 is more resilient. Although the vitality of population flow between cities is relatively low in 2020, its overall network response is relatively stable. Conversely, the urban population flow network in 2021 exposed regional vulnerability and resource disequilibrium under the background of large-scale population mobility.

Generally speaking, in the population flow network during the SFTR, the enhancement of population mobility intensity promotes the communication and cooperation between cities, as well as accumulates the potential pressure and uncertainty. Furthermore, Jingzhou, Huanggang, Yichun, Xiaogan, etc., which are closer to the center of the regional network, have undertaken the reconfiguration of most urban elements after the core cities were attacked. They perform the potential functions of population collection and distribution and maintaining the stability of the network

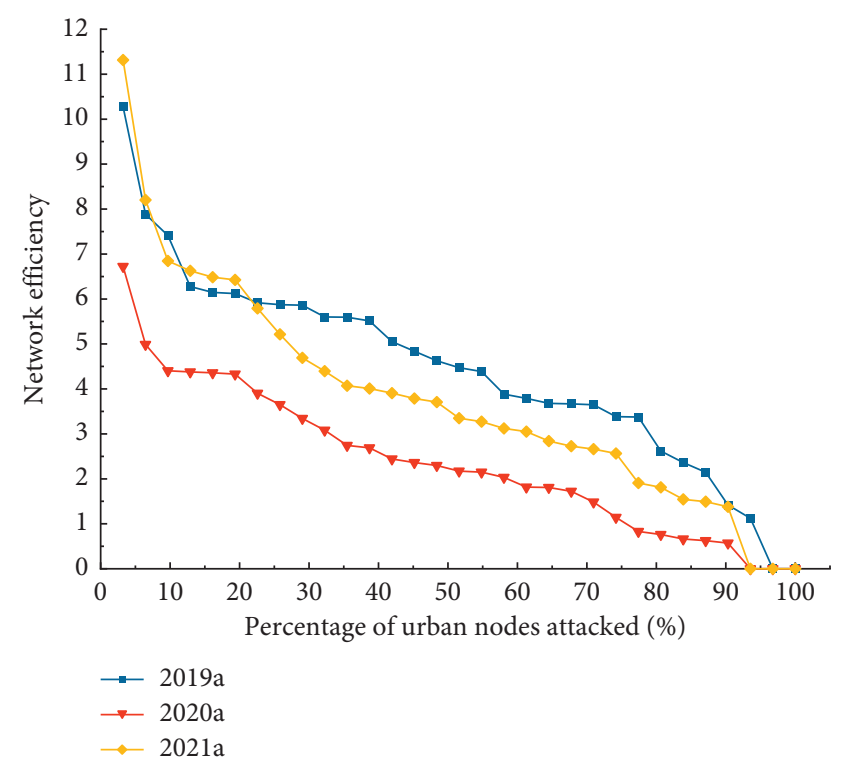

FIgURE 8: The changes in the network efficiency after attacking urban nodes in 2019, 2020, and 2021.

structure. Therefore, while emphasizing on the development and construction of core cities, we should pay more attention to the construction and support of such in-between cities in the transportation hubs, resource allocation, and information sharing to improve the resilience of regional urban systems.

\section{Discussion}

Increasingly, interweaving physical infrastructure and virtual networks present a more abundant connotation. Research on regional resilience and dynamic process of urban systems has been paid more and more attention by the scholars of geography and other fields. However, due to limitations in data acquisition, most of the related studies just stay in static expresses of characteristics. To some extent, the characteristics of the dynamic and complex population mobility networks have been neglected. What's worse, in the process of economic and technological globalization, the long-term chronic stress and short-term effects faced by regions and cities have become more prominent. Cities as multiagent systems play an indispensable role in functional integration and organizational management in regions [35]. Population floating, as the main carriers of regional intercity flow elements, the quantification and analysis of the dynamic properties of which is a powerful basis for understanding regional development. Therefore, it is urgent to measure and analyze the regional urban networks from the perspective of spatiotemporal evolution and resilience.

In this study, from the characteristics of population flow of the urban agglomeration in the middle reaches of the Yangtze River, it is revealed that the travel trends of residents during SFTR in 2019, 2020, and 2021 are consistent, that is, it increased with the approaching and ending of the Spring Festival Holiday and showed a lower trend on the day of the Spring Festival. The result is similar to the research-related 
results in other regions. For example, Lai and Pan [30] analyzed the characteristics and spatial pattern of population flow among cities in China in 2018 based on Tencent migration data and found that the population travel during SFTR showed certain regularity. In addition, from the spatiotemporal patterns and node criticality of the population flow networks, even if Wuhan was quarantined on January 23,2020 , the population flow barycenter was only more inclined to Changsha and the corridor cities between them. In other words, Nanchang, the third largest city in the urban agglomeration, still had not played a significant role. Among the popular migration routes of the urban agglomeration, Nanchang did not take a place. With the rise of its urban functional status, the situation of tripartite confrontation in the region has slightly appeared. On the one hand, the urban agglomeration in the middle reaches of the Yangtze River is the largest cross-region urban agglomeration in China. The distance between the core cities is more than $250 \mathrm{~km}$, which is much higher than $100-200 \mathrm{~km}$ between the core cities of the top three urban agglomerations (Beijing-Tianjin-Hebei, Yangtze River Delta, and Pearl River Delta). Among the 31 node cities in the study area, Xiangyang and Shangrao are the cities with the farthest distance, which is about $700 \mathrm{~km}$. On the other hand, the economic capacities of Wuhan, Changsha, and Nanchang has a certain gap and are far lower than the core cities of large urban agglomerations, such as Beijing, Shanghai, Guangzhou, Shenzhen, and so forth. Therefore, the integrative development of the urban agglomeration poses a severe test to both the economic power and radiation capacity of core cities. This is also the reason for the great differences in the development of the provinces within the urban agglomeration. What's more, the results of regional resilience via interruption simulations reveal that large-scale population floating may aggravate the instability of the regional urban network. Especially for the core cities and their corridor cities, a reasonable planning and development of their urban transportation infrastructure systems is the prerequisite to ensure regional stability.

Although an important planning area in the national development strategy, the urban agglomeration in the middle reaches of the Yangtze River, for which the government has given great support in development policies and funds, its inherent urban contact still restricts the integrated and coordinated development of the region. As we have shown in the results of this study, it is difficult for the population migration within the urban agglomeration based on Autonavi migration data to cross the provincial boundaries and connect the core cities. Therefore, in order to promote and guarantee the highly efficient flow of various elements within the urban agglomeration, especially the flow of materials and resources, we try to put forward strategies and suggestions from the perspective of urban connections for the reference of government managers and decisionmakers. First, the government should focus on optimizing the spatial patterns among the core cities of the urban agglomeration. A stable "triangle" top-level network spatial structure with Wuhan, Changsha, and Nanchang as the vertices could be formed in the region, which can ensure the cross-regional cooperative transportation capacity of material resources to a certain extent. In recent years, regional uncertainty risks (such as floods, mud-rock flows, infectious diseases, etc.) have occurred increasingly. Once a core city is in a serious disaster area, its abilities in personnel coordination and resource dispersion may be slightly insufficient. Then, as the nearest neighbor and the most powerful helper, the core cities in the adjacent regions play a leading role in resource allocation and time compression. Therefore, the strong connection between core cities can not only soften the inherent relationships within the region but also enhance regional resilience. Second, the radiation driving role of core cities should be further stimulated. Wuhan, Changsha, and Nanchang, as regions with significant concentrations of various elements at the present stage, although having the highest centrality and power within the provincial scope, their radiation driving abilities to the surrounding subtier cities are still inadequate. We should make more full use of their own advantages to spread its innovative resources such as technology, talents, and information to surrounding cities. By strengthening relationships between adjacent regions and promoting differentiation constructions, it may be possible to preferentially improve the local interaction abilities to transform "weak connections" into "strong connections", and thus drive the development of the overall urban agglomeration. Third, an increasingly elaborate regional cooperation mechanism, a flat network development mechanism with complementary functions and cross-regional cooperation, should be constructed to guide the transformation of the jurisdiction based on the hierarchical system to the network system. Province level connectivity routes across regions could be enriched by breaking administrative barriers. In addition, it should be oriented by the urban agglomeration as a whole, opening up a new prospect for high-quality synergetic development of the region. Meanwhile, urban hierarchies within the urban agglomeration should also be valued to avoid the occurrence of phenomena such as "valuing the core, ignoring the edges". Finally, timely revision and update of emergency plans and disaster mitigation measures are extremely important to safeguard regional resilience. For instance, when a largescale or sudden population migration occurs in a city, there should be correspondingly adequate prediction and response capacities to ensure the safe and sustainable working of the region. On the one hand, core cities should minimize the possibility of node failures. It can protect urban node security by strengthening emergency system construction and risk preparedness mechanisms. On the other hand, edge cities should aim at boosting the node's resilience to the risks, facilitating the circulation of elements and elevating node centrality.

Inevitably, there are some limitations in data acquisition, such as lag in socioeconomic statistics, confidentiality strategies of related sectors, etc. We extracted the scale of regional population floating using Autonavi migration data; however, it is still difficult to obtain the accurate number of passengers. The population flow networks constructed herein greatly presented the population floating patterns on the highway, which has obvious short-distance 
transportation characteristics, even during the Spring Festival travel rush [25]. However, as a part of population mobility, the overall and comprehensive relationship of population mobility between cities still needs more data to characterize. In addition, the specific reasons resulting in the abnormal population flow of urban agglomeration in the middle reaches of the Yangtze River were not further analyzed because they involve many aspects and are extremely complex. What's worse, although some other scholars have used multisource big data to estimate the migration population [36], the number of floating populations in a region itself is unstable, so there is also a larger deviation. In future relative research, the evolution and resilience of population flow networks with multisource big data based on long time series could be a promising challenge, especially for combining local and global interactions. Furthermore, the factors driving the abnormal flow of population and the influence mechanism of network structure resilience needs to be further revealed and discussed.

\section{Conclusion}

In this study, the spatiotemporal characteristics and resilience of population flow networks of urban agglomeration in the middle reaches of the Yangtze River during the Spring Festival travel rush in 2019, 2020, and 2021 were analyzed and evaluated. In the first place, Autonavi migration data (a total of 120 days) were obtained from the Autonavi big data platform based on the LBS technology to construct the regional urban networks. Second, drawing on complex network theory and methods, we analyzed the characteristics of the urban network structure during the SFTR in each period at the population flow distribution levels, the popular population floating routes, the integration scale of population floating, and the criticality of urban nodes. What's more, the resilience of the urban network structure was estimated based on node failures and attacks. In practice, this study can provide a branch for guiding rational population flow and planning of the regional urban system, thereby enhancing the further resilient development of the region. The main conclusions are as follows:

(1) During the 40-day Spring Festival travel rush, the changing trends of population flow in the three periods are consistent, showing travel peaks as the festival draws near and ends, with the highest travel peak being the last day of the Spring Festival Holiday. The population floating scale in 2020 and 2021 shows significant fluctuations before and after the Spring Festival. After the Spring Festival in 2020, the epidemic prevention and control policies restricted the travel of a large number of people; therefore, the population migration showed a downward trend. The SFTR in 2021 was a critical period for epidemic prevention and control. To curb the spread of the coronavirus, under the appeal of the China government in advocating off-peak travel and stay put during the Spring Festival, the population migration before the Spring Festival was relatively conservative, and its migration willingness was only comparable to the actual migration scale in 2019, and also lower than that in the same period of other years. However, as prevention and control work in an orderly way, the population migration after the Spring Festival immediately rebounded to the level of 2019, and surpassed that in 2019 after the Spring Festival Holiday.

(2) The intercity population flow network based on the Autonavi actual migration scale of the three periods during the SFTR has obvious differences in connection intensity and hierarchical distribution. It is reflected in the overall attenuation after the impact of the epidemic and the continuous improvement under effective prevention and control. Moreover, there is an obvious clustering phenomenon within each province of urban agglomeration, that is, under abnormal disturbance and influence, the urban cluster structure is still highly consistent. The popular migration routes in 2019 and 2021 mainly focused on Wuhan, which was characterized by the agglomeration of surrounding cities to core cities. In 2020, Changsha became the first popular distribution node. In addition, it is characterized by the migration trend from the core provincial capital cities to the surrounding small cities.

(3) The population floating intensity of most urban nodes presents asymmetric characteristics during the SFTR from the perspective of the node symmetry index. On the whole, the siphon effect of the core cities is gradually increasing, while the neighboring cities are the primary objects. According to the criticality of nodes, Nanchang ranked third after Wuhan and Changsha, but its core role has not been highlighted, indicating that a tripartite confrontation in the urban agglomeration gradually forms. Besides, Jingzhou, Xiaogan, Huanggang, Yichun, and other cities should not be underestimated in their status in the regional urban network.

(4) The disturbances of a city node may lead to a decrease in the overall transmission efficiency of the regional network. The higher the core position of a node is, the more significant the decline in the network efficiency. In addition, although the intensity of population flow during the SFTR in 2021 is higher than that in 2019 and 2020, the instability of the network structure is higher, and the potential risks and pressures are even greater. Therefore, attention should also be paid to the construction and development of the peripheral cities in the aspects of resource allocation and infrastructure arrangement to improve the regional resilience and ability to respond to disturbances.

\section{Data Availability}

Autonavi migration data can be obtained from the Amap traffic big data released by Autonavi Company (https://trp. autonavi.com/migrate/page.do). 


\section{Conflicts of Interest}

The authors declare that there are no conflicts of interest regarding the publication of this paper.

\section{Acknowledgments}

This study was funded by the National Natural Science Foundation of China (Nos. 42071216 and 41661025).

\section{References}

[1] M. Castells, "Grassrooting the space of flows," Urban Geography, vol. 20, no. 4, pp. 294-302, 1999.

[2] S. M. Li, "Population migration, regional economic growth and income determination: a comparative study of dongguan and meizhou, China," Urban Studies, vol. 34, no. 7, pp. 999-1026, 1997.

[3] J. Pan and J. Lai, "Spatial pattern of population mobility among cities in China: case study of the National Day plus Mid-Autumn Festival based on Tencent migration data," Cities, vol. 94, pp. 55-69, 2019.

[4] S. Chen, J. Yang, W. Yang, C. Wang, and T. Bärnighausen, "COVID-19 control in China during mass population movements at New Year," The Lancet, vol. 395, no. 10226, pp. 764-766, 2020.

[5] H. De Haas, "Migration and development: a theoretical perspective," International Migration Review, vol. 44, no. 1, pp. 227-264, 2010.

[6] J. Liu, J. Y. Hao, Y. Y. Sun, and Z. W. Shi, "Network analysis of population flow among major cities and its influence on COVID-19 transmission in China," Cities, vol. 112, Article ID 103138, 2021.

[7] H. Bherwani, S. Anjum, S. Kumar et al., "Understanding COVID-19 transmission through Bayesian probabilistic modeling and GIS-based Voronoi approach: a policy perspective," Environment, Development and Sustainability, vol. 23, no. 4, pp. 5846-5864, 2021.

[8] Z. Sun, H. Zhang, Y. Yang, H. Wan, and Y. Wang, "Impacts of geographic factors and population density on the COVID-19 spreading under the lockdown policies of China," The Science of the Total Environment, vol. 746, Article ID 141347, 2020.

[9] C. Zhao, N. Li, and D. P. Fang, "A conceptual framework for modeling critical infrastructure interdependency: using a multilayer directed network model and targeted attack-based resilience analysis," Computing in Civil Engineering, pp. 347-354, 2015.

[10] Y. J. Shi, G. F. Zhai, L. H. Xu et al., "Assessment methods of urban system resilience: from the perspective of complex adaptive system theory," Cities, vol. 112, Article ID 103141, 2021.

[11] R. Boschma, "Towards an evolutionary perspective on regional resilience," Regional Studies, vol. 49, no. 5, pp. 733-751, 2015.

[12] C. S. Holling, "Resilience and stability of ecological systems," Annual Review of Ecology and Systematics, vol. 4, no. 1, pp. 1-23, 1973.

[13] M. Bruneau, S. E. Chang, R. T. Eguchi et al., "A framework to quantitatively assess and enhance the seismic resilience of communities," Earthquake Spectra, vol. 19, no. 4, pp. 733-752, 2003.

[14] Y. Wang, J. Zhan, X. Xu, L. Li, P. Chen, and M. Hansen, "Measuring the resilience of an airport network," Chinese Journal of Aeronautics, vol. 32, no. 12, pp. 2694-2705, 2019.
[15] M. Janić, "Reprint of "Modelling the resilience, friability and costs of an air transport network affected by a large-scale disruptive event"," Transportation Research Part A Policy and Practice, vol. 81, pp. 77-92, 2015.

[16] K. Anand, P. Gai, S. Kapadia, S. Brennan, and M. Willison, “A network model of financial system resilience," Journal of Economic Behavior and Organization, vol. 85, pp. 219-235, 2013.

[17] Y. T. Ding, M. Zhang, S. Chen, and R. Nie, “Assessing the resilience of China's natural gas importation under network disruptions," Energy, vol. 211, Article ID 118459, 2020.

[18] M. Li, H. Wang, and H. Wang, "Resilience assessment and optimization for urban rail transit networks: a Case Study of Beijing Subway Network," IEEE Access, vol. 7, pp. 7122171234, 2019.

[19] N. Mou, S. Sun, T. Yang et al., "Assessment of the resilience of a complex network for crude oil transportation on the Maritime Silk Road," IEEE Access, vol. 8, pp. 181311-181325, 2020.

[20] X. H. Feng, C. L. Xiu, L. M. Bai, Y. X. Zhong, and Y. Wei, "Comprehensive evaluation of urban resilience based on the perspective of landscape pattern: a case study of Shenyang city," Cities, vol. 104, Article ID 102722, 2020.

[21] X. Liu, Z. Neal, and B. Derudder, "Featured graphic. City networks in the United States: a comparison of four models," Environment and Planning A, vol. 44, no. 2, pp. 255-256, 2012.

[22] A. Li, N. Mou, L. Zhang, T. Yang, W. Liu, and F. Liu, “Tourism flow between major cities during China's national day holiday: a social network analysis using weibo check-in data," IEEE Access, vol. 8, pp. 225675-225691, 2020.

[23] S. Wei and L. Wang, "Examining the population flow network in China and its implications for epidemic control based on Baidu migration data," Palgrave Communications, vol. 7, Article ID 145, 2020.

[24] X. Wei and X. A. Yao, "Constructing and analyzing spatialsocial networks from location-based social media data," Cartography and Geographic Information Science, vol. 48, no. 3, pp. 258-274, 2021.

[25] R. Zhang, J. H. Pan, and J. B. Lai, "Network structure of intercity trips by Chinese residents under different travel modes: a case study of the Spring Festival travel rush," Complexity, vol. 2021, Article ID 1283012, 2021.

[26] Q. S. Gu, H. P. Zhang, M. Chen, and Y. Xie, "Hierarchical network structures and regional differentiations of tourist source destinations of Nanjing based on cellular signaling data," Scientia Geographica Sinica, vol. 39, no. 11, pp. 17391748, 2019, (in Chinese).

[27] N. Limtanakool, M. Dijst, and T. Schwanen, "A theoretical framework and methodology for characterising national urban systems on the basis of flows of people: empirical evidence for France and Germany," Urban Studies, vol. 44, no. 11, pp. 2123-2145, 2007.

[28] B. J. L. Berry, "Cities as systems within systems of cities," Papers of the Regional Science Association, vol. 13, no. 1, pp. 146-163, 1964.

[29] H. Ishii, R. Tempo, and E.-W. Bai, "A web aggregation approach for distributed randomized PageRank algorithms," IEEE Transactions on Automatic Control, vol. 57, no. 11, pp. 2703-2717, 2012.

[30] J. B. Lai and J. H. Pan, “China's city network structural characteristics based on population flow during Spring Festival travel rush: empirical analysis of "Tencent Migration" big data," Journal of Urban Planning and Development, vol. 146, no. 4, Article ID 04020018, 2020. 
[31] S. S. Chopra and V. Khanna, "Understanding resilience in industrial symbiosis networks: insights from network analysis," Journal of Environmental Management, vol. 141, pp. 86-94, 2014.

[32] X. Li and R. Xiao, "Analyzing network topological characteristics of eco-industrial parks from the perspective of resilience: a case study," Ecological Indicators, vol. 74, pp. 403-413, 2017.

[33] S. Latora and A. Marchiori, "Attack vulnerability of scale-free networks due to cascading breakdown," Physical Review, vol. 70, no. 3, pp. 5101-5114, 2001.

[34] C. J. Fan, T. M. Cai, Z. Y. Gai, and Y. R. Wu, "The relationship between the migrant population's migration network and the risk of COVID-19 transmission in China-empirical analysis and prediction in prefecture-level cities," International Journal of Environmental Research and Public Health, vol. 17, no. 8, Article ID 2630, 2020.

[35] Y. Wei, J. E. Wang, W. Song, C. L. Xiu, L. Ma, and T. Pei, "Spread of COVID-19 in China: analysis from a city-based epidemic and mobility model," Cities, vol. 110, Article ID 103010, 2020.

[36] Z. Liu, J. L. Qian, Y. Y. Du, J. W. Yi, and Y. Sun, "Multi-level spatial distribution estimation model of the inter-regional migrant population using multi-source spatio-temporal big data: a case study of migrants from Wuhan during the spread of COVID-19," Journal of Geo-information Science, vol. 22, no. 2, pp. 147-160, 2020, (in Chinese). 